\title{
Sitting Still: Seat Design for a New Head-Only MRI Scanner
}

\section{Abstract}

Magnetic resonance imaging is the gold standard in medical practice for tissue imaging. However, current devices are expensive and require users to be in a supine position during the scanning procedure, with users often reporting claustrophobia and anxiety. This study reports on the design of a patient handling system for a new kind of head-only MRI scanner, which allows users to be seated. This poses a unique challenge in seat design: the design should allow the user to be still to attain accurate MRI scans. We investigate the aesthetics of interaction in this complex dynamic system to yield a comfortable user experience. We followed a human-centred design process comprising two main design phases. An experimental setup was used to evaluate possible upright positions of the user. A refined functional prototype was consequently built and tested with healthy participants. Our results indicate that the position required to sit in a lounge chair is most comfortable and allows subjects to remain still for an extended time period. Our chair prototype provided sufficient support during the 20 minute procedure but needs further refinement to improve the user experience and usability of the system.

\section{Keywords}

MRI, medical design, seating design, design prototype, user experience

\section{Introduction}

Magnetic resonance imaging (MRI) is a tremendously versatile technique for tissue imaging, with applications as diverse as cardiac health monitoring through to brain tumour detection. MRIs are used in five major anatomical regions: central nervous system, musculoskeletal, body, cardiovascular, and breast imaging [1]. To obtain accurate images of the human body, existing MRI scanners require the patient to remain motionless in a confined horizontal space. The enclosed space and extended time period that the patient is asked to remain still can cause enormous anxiety prior to and during the scan [2]. Techniques used in clinical practice that aim to reduce anxiety and motion during the scan include the provision of relevant information, playing music during the procedure [3]-[5], providing a mirror to look outside the bore [6] and adding foam pads [4], [7]. However, the process can be extremely challenging for patients. A feeling of lack of control [8] as well as lying on a stretcher during the procedure [4] have been identified as negative factors in the user experience and aesthetics of interaction. The development of a seated, head-only imaging system dedicated to brain imaging, which is one of the most common applications for MRIs, would offer a less restricting scanning procedure. It could furthermore contribute to a feeling of control due to the upright position and being able to look outside 
the magnet using a small window. Such a novel system could be transportable, increasing access for a wider population, especially people living in isolated areas. The proposed new kind of MRI scanner is currently under development by a world-leading team of MRI experts [8].

We report in this study on the design of the seating user-experience from a human-centred design approach [9] and with consideration to the aesthetics of interaction [10].

\section{Background}

Patients who are scanned with an $\mathrm{MRI}$ are required to lie on a stretcher, which is then moved inside the bore of the magnet. There are few MRI applications that allow a prone instead of the standard supine position such as the MRI breast scanner [11]. Further variations allow a seated position [12] or offer detachable and MRI-compatible seating elements to scan patients that cannot be imaged in the traditional position, or to gather specific imaging that requires gravity, for example, spine scans [13]. Extremity MRI systems [14] seem to provide a comfortable experience for the patient but do not allow a stable and upright position for the patient's head, which is one of the technical constraints that our design needs to address.

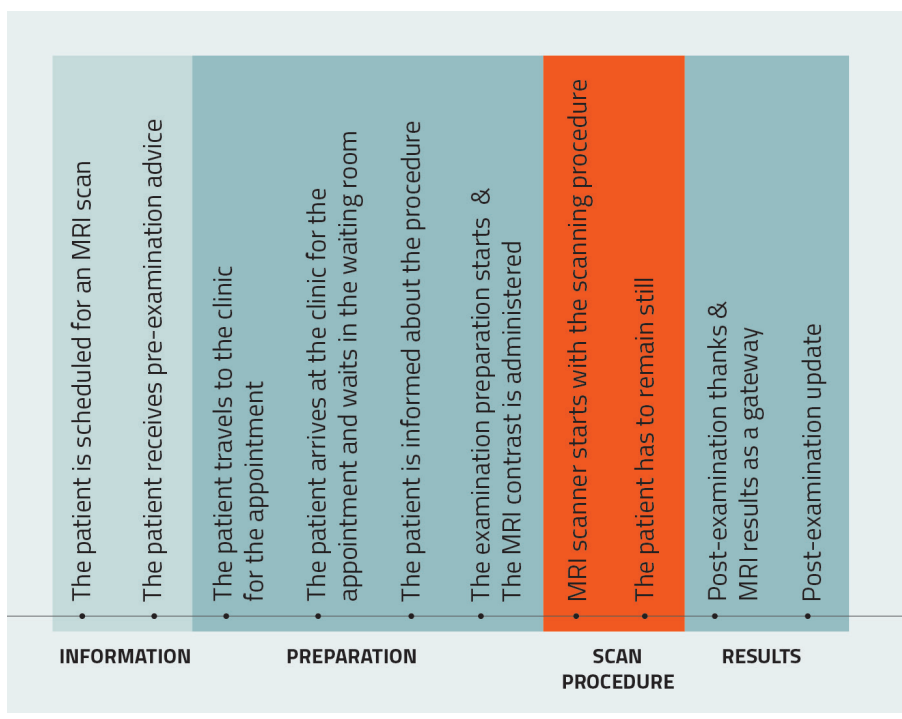

Fig. 1. Milestones of the user experience. We focus in this study on the orange part of the experience.
The aesthetics of interaction refers to "the way an object speaks to us, calls us, affords us, puts us into contact with others, is meaningful to us" [10]. Aesthetics of interaction is experienced at the ongoing interaction space where the physical characteristics of the product and the information it conveys (functional, augmented, inherent) meet and converse back and forward with the user's context (general knowledge, aesthetic preferences, cognitive and affective states, personality, motivation) and sensory-motor system [15]. We reviewed design variables of MRI systems and other industrial chair designs, medical chairs and automotive car seat designs that would allow an upright position. Currently no other seating system exists that could be used as part of a head-only MRI scanner, let alone one that addresses the aesthetics of interaction. Interestingly, automotive car seat designs pay much more attention to the aesthetics of interaction than other reported medical device designs, by trying to create a beautiful experience as well as addressing the highly demanding technical constraints of car seat design.

A number of studies focus on the experience of MRI systems and user experience of patients as well as technical staff [2], [4], [6], [8], [16]-[20], but fail to mention explicitly how to design for the aesthetics of interaction. We developed a customer journey map based on these studies, see Figure 1 for an overview of the main milestones. The studies indicate that the MRI user experience is often extremely challenging, see Table 1 for details relating to the scanning process, which is the main focus of this study. It has been reported that up to $37 \%$ of patients experience a moderate to high level of anticipatory anxiety [2]. The main contributor seems to be a feeling of claustrophobia [17] and associated negative emotions already start to set in before the actual scanning procedure takes place [19]. The anxiety can even manifest itself after the scan affecting the daily routine of the patients [17]. It has been pointed out that anxiety and claustrophobia are strongly influenced by a feeling of suffocation, ideas of control and the potential for harm to be caused by the machine [8]. Our new design system aims to address the aesthetics of interaction by providing elements that evoke a feeling of control to improve the MRI user experience and remind the user of similar systems 
they are familiar with. In particular, we took car seat design as an area to draw inspiration from for comfort, familiarity and aesthetics of interaction.

\section{Aim}

The aim of this study was to suggest an optimal upright seating position for a head-only MRI scanner, which contributes to an improved MRI user experience while securing minimal motion of the user in combination with a head restraint system we will describe in later work.

\section{Study Context}

This study (funded by the National Institutes of Health (USA) and Kiwinet (NZ)) is part of a multi-institutional research project taking place in the United States, Brazil and New Zealand. The novel head-only MRI system is currently under development and could therefore not be used as part of our testing protocol. However, we used a physical non-functional representation of the scanner bore as part of our user testing sessions. We report in this paper specifically on the design of the seating for the new MRI system. The study was carried out by a team of industrial designers with experience in developing medical technology and a technical expert in the development of MRI magnets.

Constraints defined by engineering and clinical factors included: to design comfortable seating; head should be aligned to the magnet bore and its movement restricted; magnet cannot be moved when positioning the patient; maximum width and height of the seating area; materials need to be hospital graded if fabric and nonmagnetic if structural. This differs from traditional seating as users are not free to move their heads.

\section{Materials and Methods}

In the context of developing new medical devices, an iterative design procedure assessed by a number of usability methods is an ideal setup as part of a humancentred design approach despite being expensive and time-consuming [9]. We followed a human-centred design approach [21] with two main design phases: 1) an initial design exploration phase to investigate possible positions of the user in a sit-up MRI scanner Table 1. Customer journey milestones

\begin{tabular}{|c|c|c|c|}
\hline Step & Positive factors & Neutral factors & Negative factors \\
\hline $\begin{array}{l}\text { Start of } \\
\text { scanning } \\
\text { procedure }\end{array}$ & $\begin{array}{l}\text { 1. music used as a form of distraction } \\
\text { 2. entering the bore feet first can } \\
\text { decrease anxiety }\end{array}$ & & $\begin{array}{l}\text { 1. lack of control over the situation } \\
\text { 2. not liking or not having any music at all }\end{array}$ \\
\hline $\begin{array}{l}\text { The patient has } \\
\text { to remain still }\end{array}$ & $\begin{array}{l}\text { 1. possibility to have a nap } \\
\text { 2. noise cancelling headphones } \\
\text { 3. mental distraction } \\
\text { 4. support from others } \\
\text { 5. rewards and/or bribes } \\
\text { 6. music } \\
\text { 7. breath holding techniques } \\
\text { 8. constant reassurance that the } \\
\text { patient is in control of the situation } \\
\text { 9. sedation } \\
\text { 10. eye masks } \\
\text { 11. physical touch by the MRI } \\
\text { technician or family member } \\
\text { 12. buzzer that allows to contact staff } \\
\text { 13. trust in staff }\end{array}$ & $\begin{array}{l}\text { 1. not all patients want to } \\
\text { actively participate in the } \\
\text { scanning process } \\
\text { 2. there can be a preference } \\
\text { to hand over responsibility } \\
\text { to expert }\end{array}$ & $\begin{array}{l}\text { 1. amount of time spent in the system } \\
\text { 2. inability to keep still } \\
\text { 3. pain } \\
\text { 4. claustrophobia } \\
\text { 5. feeling unable to breathe properly } \\
\text { 6. panic symptoms } \\
\text { 7. threat to self-control } \\
\text { 8. enforced nature of having to remain motionless } \\
\text { 9. desire to scratch an itch } \\
\text { 10. thoughts about the (negative) consequences } \\
\text { 11. having panic symptoms } \\
\text { 12. lying on a hard bed } \\
\text { 13. temperature is too hot or too cold } \\
\text { 14. thoughts about the potential harm caused by the MRI } \\
\text { machine. }\end{array}$ \\
\hline
\end{tabular}


through observation, idea generation and low fidelity prototyping; and 2) a refined fully functional prototype evaluated by healthy participants.

\subsection{Design Development Process}

In a first step, we used an experimental design process to investigate patient positions in the novel MRI scanner that would allow an upright and stable position of the head for up to 30 minutes. A low fidelity prototype [22] was used to investigate where support needs to be provided in a seated position, within the specific constraints provided by the engineering team. We used pillows, a raised horizontal platform, cardboard boxes and polyurethane foam sheets to investigate how support may facilitate motionless yet comfortable sitting positions.

In a second step, we used expansive design concepts [21] exploring different designs and positions of the chair in the overall system based on the initial results. We followed a systematic approach whereby we assessed the design concepts against a list of design criteria [23] developed for this project and experience prototyping [24] to refine the design concepts until the prototype had reached a level where it could be tested with participants. A full scale of the chair was produced using a foam core and fibreglass laminate to provide the required stiffness and robustness. Adjustable upholstery was provided to the participants using a selection of different foam thicknesses. Similarly, elements for the lumbar region and movable inserts for the hip area were used in the assessment process.

\subsection{Evaluation}

A combination of different evaluation methods is helpful to determine a range of usability issues [25]. We chose a multi-method approach in the form of an ergonomic analysis [26] and formative usability testing [26] with participants. Even though the role of good design is recognised in the context of developing medical devices, it seems that ergonomic principles are often underused [9]. We employed standard data sets of human dimensions [27] and results of a study on overweight populations [28] for the ergonomic analysis process. An additional qualitative scale of comfort and discomfort, which was adapted from Helander and Zhang's chair comfort scale [29], was used as part of our testing protocol with healthy participants to confirm the results of the ergonomic analysis process. The aim of our formative evaluation process with a full-scale prototype was not the collection of quantitative data for comparison, but instead to refine the design [30]. We took the participants through a simulation of an MRI examination and asked them to remain as motionless as possible in the MRI bore during the 20 minute testing. The testing included the sound of an MRI scanner, arm movements to evaluate the degree of movement tolerable and semi-structured questions about comfort, discomfort and feelings of anxiety in regards to the chair and overall system to address aesthetics of interaction. Participants could leave the system at any stage. We used the system usability scale (SUS) [31], and an MRIAnxiety Questionnaire [32], which measures anxiety and relaxation during the MRI examination as part of the evaluation process. We used a 10 points Likert scale as part of the questionnaire. The testing was video and audio recorded for later analysis.

\subsection{Recruitment}

This study was approved by the human ethics committee of Victoria University of Wellington (VUW). Participants provided informed written consent before taking part in the testing. We recruited healthy participants through VUW. Ideal sample size suggestions for usability testings vary between 3 to 20 participants with 5-10 participants being a sensible approach for most studies [33]. Four participants took part in our first design experiments and seven in our formative usability testing.

\subsection{Analysis}

The photos, videos, notes of observations and semistructured interviews were analysed using thematic analysis in a deductive form [34]. Thematic analysis is used to identify, report, and analyse patterns and themes within the data. Themes were generated by the first author and reviewed by the remaining authors. The initial themes were translated into design criteria [23] as per Table 2 below. The design criteria were used at different stages of the research and design process to systematically assess the design concepts and refine them. The results of the scales and the questionnaire were analysed using descriptive statistics [35]. 


\begin{tabular}{|l|l|l|}
\hline & Criteria description & Based on \\
\hline 1 & $\begin{array}{l}\text { Chair fits below the MRI } \\
\text { scanner }\end{array}$ & Physical project constraints \\
\hline 2 & $\begin{array}{l}\text { Width and height fits the } \\
5 \text { th to 95th percentile }\end{array}$ & Literature: Human factors \\
\hline 3 & $\begin{array}{l}\text { Positions the user } \\
\text { correctly in the middle of } \\
\text { the bore }\end{array}$ & MRI usability constraints \\
\hline 4 & $\begin{array}{l}\text { Minimises movement of } \\
\text { the user }\end{array}$ & MRI usability constraints \\
\hline 5 & $\begin{array}{l}\text { MRI safe materials and } \\
\text { medical appearance }\end{array}$ & MRI usability constraints \\
\hline 6 & $\begin{array}{l}\text { Reduces feelings of } \\
\text { control and anxiety }\end{array}$ & $\begin{array}{l}\text { Literature: MRI user } \\
\text { experience }\end{array}$ \\
\hline 7 & $\begin{array}{l}\text { Allows arm movement of } \\
\text { the user }\end{array}$ & $\begin{array}{l}\text { Literature: MRI user } \\
\text { experience }\end{array}$ \\
\hline 8 & $\begin{array}{l}\text { Easy entering and leaving } \\
\text { of the magnet more }\end{array}$ & Physical project constraints \\
\hline
\end{tabular}

Table 2. Assessment of design concepts against design criteria

\section{Results}

\subsection{Design Development Process}

Experimental Design. Four participants took part in the initial phase of our experimental setup involving the use of low fidelity prototypes to test possible comfortable positions that ensure a straight and stable position of the head. Two participants were female and two were male. Their age range was between 22-23 years. Participants indicated that a position required to sit on a chair is the most comfortable for an extended period of time in the given physical constraints of this research. The participants mentioned that support is required for the lower back and the regions around the lumbar, neck, under the legs and buttocks to ensure comfort. Armrests and support around the thighs were identified as unnecessary. Participants acknowledged a requirement of softening of the seating-pan edge to reduce discomfort. All participants acknowledged a need for leg support however results were inconclusive as to the degree of support needed.

Expansive Design Concepts. For phase 2, we developed a range of concepts based on the results of our low fidelity prototyping. The concepts were then assessed against the updated design criteria detailed in Table 2.

Full-size Prototype. The findings suggest a position similar to a car seat or lounge chair may be appropriate to facilitate head movement restraint. This gave us the opportunity to address the aesthetics of interaction similarly to (and expand on) the car seat industry. The final chair design consisted of a seating pan and a separate, moveable backrest, see Figure 2. The chair was positioned on an electric lift column that allowed the user to be positioned correctly inside the magnet bore and could be controlled by the participant or by a research assistant. This approach was chosen based on the feeling of lack of control that multiple studies indicated. The chair prototype was tested with a physical representation of the final magnet bore and frame. We used an air ventilation system that ended just below the bore entrance, which could be regulated in terms of its intensity to provide sufficient air flow. For reference, the seat and upholstery are coloured black to assist in motion tracking of in-scan participant
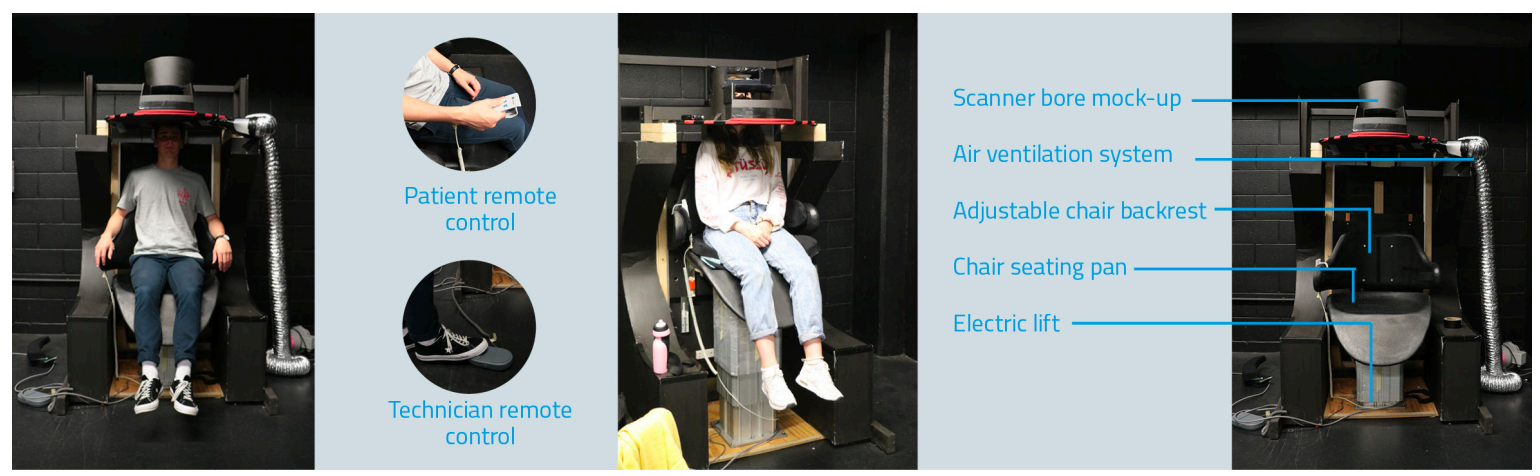

Fig. 2. Fully functional test rig with removable lumbar support and upholstery inserts. 
movement, which we are planning to carry out in the second stage of this research to confirm that our system minimises head movement to under $1 \mathrm{~mm}$. Seven participants took part in the testing. Two were female and five were male. Participants were between 20-58 years and had a height between $168-190 \mathrm{~cm}$. Their weight ranged between $50-90 \mathrm{~kg}$.

System Usability and User Experience. The results of the SUS indicated a high degree of overall usability of the system, see Table 3. However, a few participants expressed a need for more relevant information on how to use the system and a low level of confidence in using the system. The results of the MRI-Anxiety Questionnaire [32] was used to evaluate factors of anxiety due to the enclosed space and prolonged time that participants had to remain still. The results suggest that participants felt mainly relaxed, however, some participants expressed concerns regarding the ability to breath; control over the situation; and the feeling of being safe, see Figure 3.

Physical Usability Issues. We informed participants before the testing that the armrests were not completely weight bearing, which caused most to use the raised edges of the seating pan to lower themselves down into the chair. Participants had no issues leaving the chair system, however, some participants noted that some of the provided larger upholstery inserts created difficulties in entering the system.

The feet, neck and shoulder region at the back were pointed out as body parts that most participants felt a moderate to high level of discomfort with. Most body parts started to feel uncomfortable over the extended period of the testing session when the participant had to remain motionless inside the magnet bore. In particular the footrest was requested to decrease physical stress on the ankles and feet while the participants were elevated off the ground. Further notable areas for improvement were:

1. The angle of the backrest should be reclined;

2. The length of the seating pan needed extension;

3. The chair seemed to be positioned too high when at the lower travel limit of the lifting column $(50 \mathrm{~cm})$; and 4. The armrests should be designed in a way so they 'hugs' the user.
Table 3. SUS value: $0=$ low and $100=$ high,$n=7$

Fig. 3. MRI Anxiety questionnaire with a 10 points Likert scale $(n=7)$

\begin{tabular}{|l|l|l|l|l|l|l|l|}
\hline Participant & 01 & 02 & 03 & 04 & 05 & 06 & 07 \\
\hline SUS value & 70 & 95 & 77.5 & 90 & 72.5 & 85 & 72.5 \\
\hline
\end{tabular}

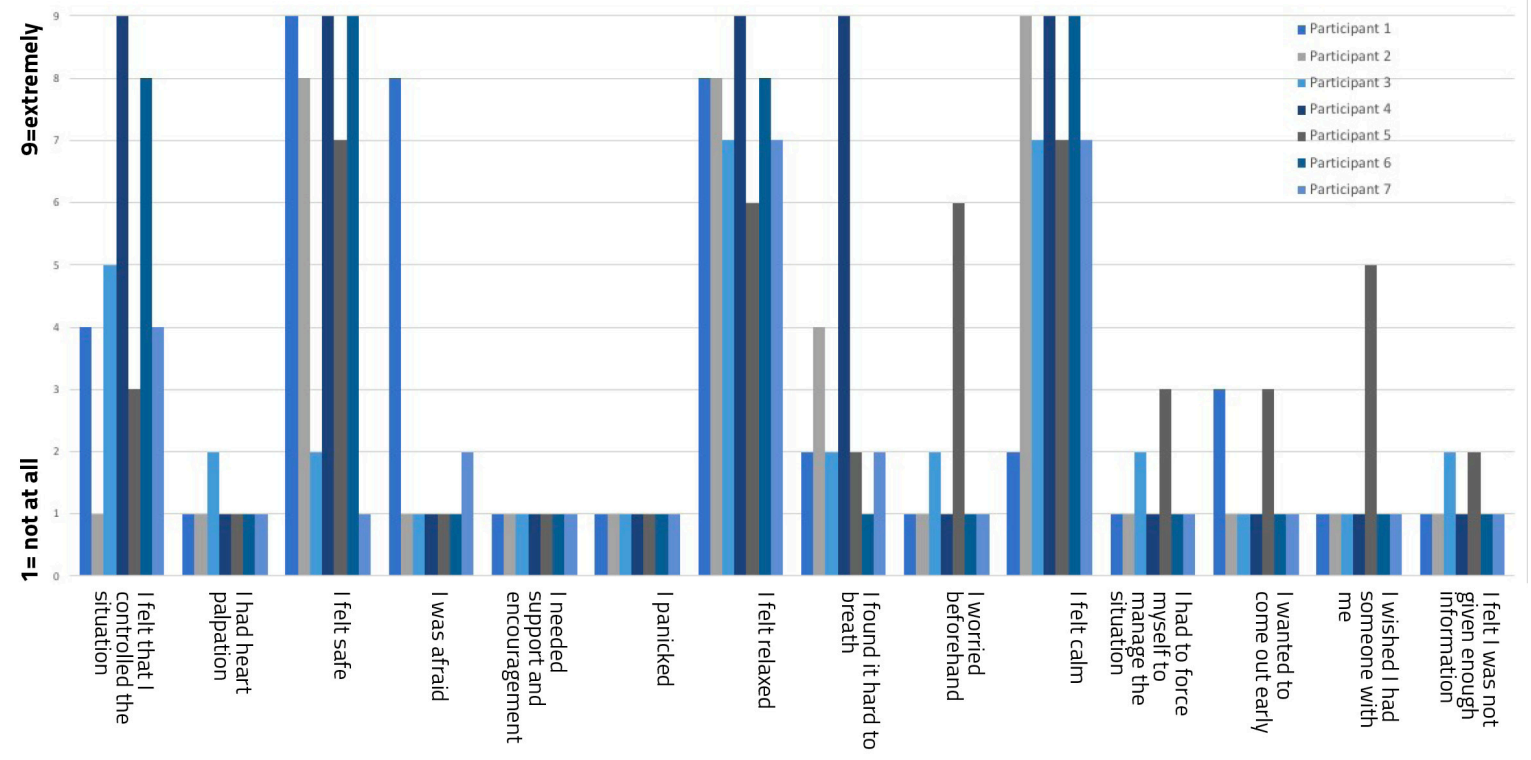




\section{Discussion}

This study reports on the development and evaluation of a chair for a sit-up, transportable MRI scanner, with the very specific requirement of keeping the user's head still for a period of time. This study is based on two design phases which informed the design of a full-scale prototype that has been tested by participants.

Our formative usability evaluation indicates that the chair provides sufficient support and comfort for a 20-minute scanning process, but requires further refinement to improve the user experience, level of comfort and usability of the system.

The SUS indicates a high level of usability of our proposed chair design. However, there were a number of observed usability issues that require further refinement. The entering and leaving of the system requires the user to bend their head in order to sit down. Arm rests were observed as an important feature that allowed a secure lowering of the user into the seating pan. The participants could use a remote control to position themselves inside the magnet bore, however, just a small number of the participants chose to do so. This might explain why some participants mentioned a lack of control over the situation, which is an important aspect in this design related to the aesthetics of interaction. The controller was difficult to use while being inside the scanner due to a lack of tactile feedback of the button that made it impossible to know which button needed to be pressed to move up or down. The participants were provided with a variety of upholstery elements to increase comfort. Some of the thicker upholstery parts compressed over the testing period consequently lowering the user further down, which meant that they either started adjusting themselves during the testing or could not look outside the scanner window anymore. The airflow was described as sufficient by some participants but not all of them. It was furthermore noted that the air felt very cold due to the high intensity. A different position of the air hose could potentially direct the air further inside the bore.

The assessment of the aesthetics of interaction produced mixed results. Car seat design was a starting point to relate users to a familiar object they would know how to interact with; this was successful in part due to the anthropometry and posture required for this project, similar to that of a car seat. However, the travel necessary for the chair to bring the patient into the magnet, our requirement to fit $95 \%$ percentile of the population, and the fact that the controls to move the chair are in a handheld remote control, meant that the aesthetics of interaction were different to a car seat.

Some of the chair features were experienced to become uncomfortable over the 20-minute testing sessions such as the 90 degree position of the seating pan in relation to the backrest that required the user to sit upright evoking lower back pain in some of the participants. A recent study focusing on the development of an extremity MRI scanner [14] suggests a reclined position for the user. The study suggests a leg rest angle between 130-148 degrees which we are going to take into consideration for the next iteration of the chair. Most of the participants recommended adding a leg rest and a foot support to avoid their feet dangling in the air during the examination. The lack of footrest evoked a high level of discomfort during our testing sessions in nearly all of the participants. This is an interesting finding as the first application of reclining chairs was for sick patients and for pregnant women to find a comfortable position without having to lie down (34). This form of chair has become associated with a level of comfort allowing the user to elevate his or her feet to increase comfort (34). Our study indicates that if the user of a chair cannot touch the ground, a leg and a footrest are essential feature to increase the user experience.

Limitations include that the initial testing had a small $(<$ $10)$ sample size, with healthy and mostly young $(<30)$ participants. The mock-up of the MRI scanner simulated user experience in a lab environment.

Based on the feedback of the participants we are currently changing the design of the chair for a second phase of usability testing. Further studies are required to confirm the usability of such a chair in a clinical environment, where patients and MRI operators are interacting with the system. However, given the high SUS scores of our current chair solution, we believe that following a final design iteration we will significantly improve subject experience during brain MRI examinations. This will have been achieved through consideration of the aesthetics of interaction in the critical chair design feature of our novel MRI system. 


\section{References}

1. Elmaoğlu, M. \& Çelik, A. (2012). MRI Handbook. Boston, MA: Springer US.

2. Katz, R.C., Wilson, L. \& Frazer, N. (1994). Anxiety and its determinants in patients undergoing Magnetic Resonance Imaging, Journal of Behaviour Therapy Experimental Psychiatry, 25(2), 131-134.

3. Törnqvist, E., Månsson, å., Larsson, E.-M. \& Hallström, I. (2006). Impact of extended written information on patient anxiety and image motion artifacts during magnetic resonance imaging, Acta Radiologica, 47(5), 474-480.

4. Funk, E., Thunberg, P. \& Anderzen-Carlsson, A. (2014). Patients' experiences in magnetic resonance imaging (MRI) and their experiences of breath holding techniques, Journal of Advanced Nursing, 70(8), 1880-1890.

5. Bolejko, A., Sarvik, C., Hagell, P. \& Brinck, A. (2008). Meeting patient information needs before magnetic resonance imaging: Development and evaluation of an information booklet, Journal of Radiology Nursing, 27(3), 96-102.

6. Carlsson, S. \& Carlsson, E. (2013). 'The situation and the uncertainty about the coming result scared me but interaction with the radiographers helped me through': A qualitative study on patients' experiences of magnetic resonance imaging examinations, Journal of Clinical Nursing, 22(21-22), 225-3234.

7. Rao, S.M., Mayer, A.R. \& Harrington, D.L. (2001). The evolution of brain activation during temporal processing, Natural Neuroscience, 4(3), 317-323.

8. Thorpe, S., Salkovskis, P.M. \& Dittner, A. (2008). Claustrophobia in MRI: the role of cognitions, Magn. Reson. Imaging, 26 (8), pp. 1081-1088.

9. Martin, J.L., Norris, B.J., Murphy, E. \& Crowe, J.A. (2008). Medical device development: The challenge for ergonomics, Applied Ergonomics, 39(3), 271-283.

10. Hummels, C. \& Overbeeke, K. (2010). Special Issue Editorial: Aesthetics of Interaction, International Journal of Design, 4(2), 1-2.

11. Konyer, N.B., Ramsay, E.A., Bronskill, M.J. \& Plewes, D.B. (2002). Comparison of MR Imaging Breast Coils, Radiology, 222(3), 830-834.

12. Fonar. (2014). Fonar upright $\AA$ multi-positionTM weightbearing MRI. Retrieved May 2019 from https://www.fonar. com/customers.htm.

13. Jinkins, J.R., Dworkin, J.S. \& Damadian, R.V. (2005). Upright, weight-bearing, dynamic-kinetic MRI of the spine: Initial results, European Radiology, 15(9), 1815-1825.

14. Jung, E.S. et al. (2019). An ergonomic user interface design for a new extremity MRI focusing on the patient chair, in Proceedings of the 20th Congress of the International Ergonomics Association (IEA 2018), 818, (pp. 139-147).

15. Locher, P., Overbeeke, K. \& Wensveen, S. (2010). Aesthetic interaction: A framework, Design Issues, 26(2), 70-79.

16. Thu, H. (Sue), Stutzman, S.E., Supnet, C. \& Olson, D.M. (2015). Factors associated with increased anxiety in the MRI waiting room, Journal of Radiology Nursing, 34 (3), 170-174.

17. Mclsaac, H., Thordarson, D., Shafran, R., Rachman, S. \& Poole, G. (1998). Claustrophobia and the Magnetic Resonance Imaging procedure, Journal of Behavioural Medicine, 21(3), 255-268.

18. Munn, Z., Jordan, Z., Pearson, A., Murphy, F. \& Pilkington, D. (2014). 'On their side': Focus group findings regarding the role of MRI radiographers and patient care, Radiography, 20(3), 246-250.

19. Hewis, J. (2015). Do MRI patients tweet? Thematic analysis of patient tweets about their MRI experience, Journal of Medical Imaging Radiation Sciences, 46(4), 396-402.

20. Munn, Z., Pearson, A., Jordan, Z., Murphy, F., Pilkington, D. \& Anderson, A. (2016). Addressing the patient experience in a magnetic resonance imaging department: Final results from an action research study, Journal of Medical Imaging Radiation Sciences, 47(4), 329-336.

21. Krogh, P.G., Markussen, T. \& Bang, A.L. (2015). Ways of drifting - Five methods of experimentation in research through design, in ICoRD'15 - Research into Design Across Boundaries Volume 1, 34 (pp. 39-50).

22. Virzi, R.A., Skolov, J.L. \& Karis, D. (1996). Usability problem identification using both low-and high-fidelity prototypes., in Conference on Human Factors in Computing Systems, Reading, Mass.

23. Rodríguez Ramírez, E. (2017). A postgraduate thesis model for research through design based on design criteria, International Journal of Design Objects, 11(4), 11-27.

24. Buchenau, M. \& Suri, J.F. (2000). Experience prototyping, in Proceedings of the 3rd conference on Designing interactive systems: processes, practices, methods, and techniques, (pp. 424-433).

25. Garmer, K., Ylvén, J. \& MariAnne Karlsson, I.C. (2004). User participation in requirements elicitation comparing focus group interviews and usability tests for eliciting usability requirements for medical equipment: A case study, International Journal of Industrial Ergonomics, 33(2), 85-98.

26. Martin, B. \& Hanington, B.M. (2012). Universal methods of design: 100 ways to research complex problems, develop innovative ideas, and design effective solutions, Digital ed. 
Beverly, MA: Rockport Publishers.

27. Tilley, A.R. \& Henry Dreyfuss Associates, Eds. (2002). The measure of man and woman: Human factors in design, Rev. ed. New York: Wiley.

28. Masson, A. (2017). Including plus size people in workplace design, Doctoral Thesis, Loughtorough University.

29. Helander, M.G. \& Zhang, L. (1997). Field studies of comfort and discomfort in sitting, Ergonomics, 40(9), 895-915.

30. Hartson, H.R., Andre, T.S. \& Williges, R.C. (2001). Criteria for evaluating usability evaluation methods, International Journal of Human Computer Interaction, 13(4), 373-410.

31. Brooke, J. (1996). SUS-A quick and dirty usability scale, Usability Evaluation in Industry, 189(194), 4-7.

32. Ahlander, B.-M., Årestedt, K., Engvall, J., Maret, E. \& Ericsson, E. (2016). Development and validation of a questionnaire evaluating patient anxiety during Magnetic Resonance Imaging: The Magnetic Resonance Imaging-Anxiety Questionnaire (MRI-AQ), Journal of Advanced Nursing, 72(6), 1368-1380.

33. Macefield, R. (2009). How to specify the participant group size for usability studies: A practitioner's guide, Journal of Usability Studies, 5(1), 34-45.

34. Braun, V. \& Clarke, V. (2006). Using thematic analysis in psychology, Qualitative Research in Psychology, 3(2), 77-101.

35. Salkind, N. (2010). Descriptive statistics, Encyclopedia of Research Design. SAGE Publications, Inc., 2455 Teller Road, Thousand Oaks California 91320 United States. 